\section{Cultural psychiatry}

\section{4}

Two-eyed seeing as a philosophy to facilitate communication between indigenous counselors and psychiatry about mind and mental health

\author{
B. Mainguy ${ }^{1 \star}$ and L. Mehl-Madrona ${ }^{2}$ \\ ${ }^{1}$ Education Division, Coyote Institute - Canada, Ottawa, Canada and \\ ${ }^{2}$ Medical Arts And Humanities Program, University of Maine, Orono, \\ United States of America \\ ${ }^{\star}$ Corresponding author. \\ doi: $10.1192 /$ j.eurpsy.2021.303
}

Introduction: The term "two-eyed seeing" is spreading across North America as a concept for explanatory pluralism. The concept was brought into academic science by Albert Marshall, a M'iqmaq from Nova, Scotia, Canada. It speaks to the idea that indigenous knowledge is an equally valid way of conceptualizing a phenomenon as is contemporary science. Marshall's famous example compares a traditional M'iqmaq story about the origins of the large tides in the Bay of Fundy with contemporary oceanographic geology findings and simulations.

Objectives: We wanted to explore how this two-eyed seeing model could be applied to mental health to facilitate a dialogue between psychiatry and traditional cultural healers.

Methods: We reviewed the existing literature on two-eyed seeing within mental health care using PubMed, IndexMedicus, OneSearch, and Google Scholar. We presented a course on two-eyed seeing for indigenous mental health services and two-eyed seeing for addressing trauma in indigenous communities and surveyed the participants about the two-eyed seeing concept. We offered this course primarily to providers within indigenous communities and also for other interested counsellors.

Results: Participants in our trainings were enthusiastic about the role of two-eyed seeing for improving communication among indigenous providers and patients and non-indigenous providers. Most indigenous counselors had not heard of two-eyed seeing and were quite enthusiastic about its affirming nature and how it gave them a basis for dialogue with non-indigenous practitioners.

Conclusions: Two-eyed seeing allows a rich dialogue between European-derived practitioners and indigenous people that enabls each to appreciate the other's perspectives, leading to greater cooperation and collaborative treatment.

Disclosure: No significant relationships.

Keywords: Indigenous people; two-eyed seeing; explanatory pluralism; cross-cultural communication

\section{6}

Group psychological intervention for people affected by conflict in Central African republic

\author{
E. Dozio ${ }^{1 \star}$ and C. Bizouerne ${ }^{2}$ \\ ${ }^{1}$ Mental Health And Care Practices, Action Contre la Faim, Paris, \\ France and ${ }^{2}$ Mental Health And Care Practices, Gender And \\ Protection, Action contre la Faim, Paris, France \\ ${ }^{*}$ Corresponding author. \\ doi: $10.1192 /$ j.eurpsy.2021.304
}

Introduction: A large part of the Central African population has been exposed to potentially traumatic events as a result of the recent conflict, which has led to the breakdown of social ties.

Objectives: Faced with this situation, the NGO Action contre la Faim proposed a psychosocial intervention aimed at helping the displaced people to reduce their psychological suffering and strengthen individual and community resilience.

Methods: After psychoeducation sessions organized in communities affected by the conflict, people identified with traumatic symptoms are invited to participate in a psychological support intervention. The protocol used is based on the Problem Management Plus (PM+), developed by the WHO. The approach was adapted in groups to reach more suffering people and also to take advantage of the group dynamic in the possibility of recovering and developing better resilience.

Results: 946 IDPs in the country's capital, participated in the group intervention led by a team of paraprofessionals. Data collected from 111 participants show that after five weeks of intervention, there was a significant reduction in post-traumatic symptoms (PCL-5) and functional impairment (WHODAS). These results were confirmed during the post-intervention evaluation four weeks later. In addiction, participants declared that they had observed effects on their ability to live together in the community and to regain social cohesion.

Conclusions: This experience gives encouraging results with regard to the feasibility and replicability of the group protocol, taking into account specific cultural and contextual adaptations.

Disclosure: No significant relationships.

Keywords: PM+; Central African Republic; Cultural Psychology; social cohesion

\section{7}

\section{Cultural competence in modern global world:} Applications for mental health

\section{R. Shilko* and L. Shaigerova}

Faculty Of Psychology, Lomonosov Moscow State University, Moscow, Russian Federation

${ }^{\star}$ Corresponding author.

doi: 10.1192/j.eurpsy.2021.305

Introduction: Cultural competence and related terms began to appear in the 1960s in the context of the development of civil rights movements in many countries. The importance of research of cultural competence among mental health professionals is raised with the globalization trends of the modern world, when the growth of ethnocultural diversity, internal and external migration, temporary movement of people lead to intensification of intercultural interaction.

Objectives: The current study aims to reveal contemporary tendencies in cultural competence understanding and development.

Methods: Theoretical analysis and systematization of research publications in order to clarify concepts, models and applications of cultural competence.

Results: The following tendencies were revealed. Cultural competence continues to attract significant attention of researchers and practitioners, especially among the mental health specialists (psychologists, psychiatrists, psychotherapists) who work with representatives of different cultures. A number of similar concepts and their components have been proposed: cultural competence, intercultural communicative competence, cross-cultural competence, cultural intelligence, cultural awareness, cultural acceptance, intercultural sensitivity, intercultural adaptation, multicultural competence, multicultural orientation. The difficulties and limitations of 